
\title{
A hybrid stochastic model of the budding yeast cell cycle
}

\author{
Mansooreh Ahmadian $\mathbb{D}^{1}$, John J. Tyson $\mathbb{D}^{2 凶}$, Jean Peccoud $\mathbb{D}^{3}$ and Yang Cao $\mathbb{D}^{1 凶}$
}

The growth and division of eukaryotic cells are regulated by complex, multi-scale networks. In this process, the mechanism of controlling cell-cycle progression has to be robust against inherent noise in the system. In this paper, a hybrid stochastic model is developed to study the effects of noise on the control mechanism of the budding yeast cell cycle. The modeling approach leverages, in a single multi-scale model, the advantages of two regimes: (1) the computational efficiency of a deterministic approach, and (2) the accuracy of stochastic simulations. Our results show that this hybrid stochastic model achieves high computational efficiency while generating simulation results that match very well with published experimental measurements.

npj Systems Biology and Applications (2020)6:7; https://doi.org/10.1038/s41540-020-0126-z

\section{INTRODUCTION}

The eukaryotic cell cycle is a complex process by which a growing cell replicates its DNA and divides into two cells, each capable of repeating the process. Progression through the cycle is controlled by networks of genes, mRNAs, and proteins, with interactions that can be modeled as chemical reaction channels. To unravel the complex dynamics of multi-scale reaction networks in higher organisms such as human cells, it is advisable to study single-cell organisms with molecular regulatory networks that are similar yet simpler. For instance, experimental studies and mathematical models of frog eggs ${ }^{1,2}$, fission yeast ${ }^{3,4}$, and budding yeast ${ }^{5,6}$ have shed light on mechanisms of cell-cycle regulation in the cells of higher organisms. Extensive experimental studies have been conducted particularly on budding yeast (Saccharomyces cerevisiae) to explore gene regulation and signaling pathways of relevance to cell growth and division ${ }^{7-9}$. Moreover, various modeling approaches, such as deterministic models ${ }^{10-12}$, Boolean networks ${ }^{13-19}$, and stochastic models ${ }^{20-26}$, have been adopted to explore the roles of different gene and protein interactions in robust progression through the cell cycle.

Among these models, a deterministic approach is most common. In this approach, the time-dependent variation of each molecular species in the biochemical reaction network is described by a nonlinear ordinary differential equation (ODE), in which the concentration of the substance is considered as a continuous quantity that evolves deterministically over time. However, the time-evolution of molecular species within the confined volume of a budding yeast cell (about $30 \mathrm{fL}$ at birth) is not deterministic. Therefore, in spite of being able to reproduce certain average characteristics of cell-cycle progression in yeast cell populations, a continuous-deterministic model cannot reproduce the cell-to-cell variability observed in wet-lab experiments $^{27,28}$. For instance, Di Talia et al. ${ }^{28}$ have reported that the coefficient of variation ( $\left.\mathrm{CV}=\frac{\text { standard deviation }}{\text { mean }}\right)$ for $\mathrm{G} 1$ time of budding yeast cells (growing on glucose) is $50 \%$.

To capture such high levels of variability, stochastic models have been built using different strategies to incorporate intrinsic and extrinsic sources of noise. In an early stochastic model of the fission yeast cell cycle proposed by Sveiczer et al. ${ }^{29}$, extrinsic noise was introduced by assuming some sloppiness in the partitioning of cell volume and nucleus volume to daughter cells at division. A later model by Steuer ${ }^{30}$ examined the roles of intrinsic noise in cell-cycle progression by adding Gaussian noise to reaction rate equations in a deterministic model. These approaches, however, do not adequately explain the root source of cell-cycle variability in yeast cells, which lies in molecular fluctuations at the level of gene expression ${ }^{31-33}$. To capture such molecular-level noise, more accurate stochastic methods are required to explicitly model fluctuations in molecular interactions. For this purpose, the best method to implement fluctuating molecular interactions is the stochastic simulation algorithm (SSA) proposed by Gillespie ${ }^{34}$. Gillespie's algorithm is a Monte-Carlo approach that numerically simulates the temporal firing of every single reaction in a chemical reaction network. An assumption of Gillespie's method is that the propensity of every reaction in the model is described by massaction kinetics. This becomes an issue for us because most deterministic models of cell-cycle regulation, such as those presented in refs $5,11,12,35$, incorporate complex rate laws including Michaelis-Menten kinetics, Hill functions, and ultra-sensitive switches. These complex phenomenological rate laws are used in deterministic models to provide sufficient nonlinearity in reaction kinetics to create bistable switches that flip on and off during progression through the cell cycle. Converting a deterministic model into a stochastic model suitable for Gillespie's SSA by 'unpacking' complex rate laws into elementary reactions is a difficult problem fraught with uncertainties ${ }^{36}$.

To address this challenge, several approaches have been tried. The simplest approach, used for example by Mura \& Csikasz$\mathrm{Nagy}^{37}$, treats all complex rate laws directly as propensity functions of reactions and then applies the SSA. This approximation is subject to considerable errors ${ }^{36,38}$. For example, Ball et al. ${ }^{21}$ found that the variability they observed in wet-lab measurements could not be generated by this greatly simplified stochastic approach, unless some unrealistic parameter values were chosen. Later Kar et al. ${ }^{20}$ tried to unpack Michaelis-Menten rate laws in a small (three-variable), deterministic model of the budding yeast cell cycle ${ }^{5}$. Unpacking resulted in a much more complicated system with 19 species and 47 reactions. Although this simple model (with only a few key cell-cycle genes) could generate noise levels that match wet-lab measurements for a few key characteristics of the cell cycle, it is not feasible (in our experience) to apply this approach to more complex models with substantially more genes and proteins. Instead, we have pursued an approach in which the molecular controls of the budding yeast cell cycle are

\footnotetext{
${ }^{1}$ Department of Computer Science, Virginia Tech, Blacksburg, VA, USA. ${ }^{2}$ Department of Biological Sciences, Virginia Tech, Blacksburg, VA, USA. ${ }^{3}$ GenoFAB Inc, Fort Collins, CO, USA.

凶email: tyson@vt.ecu; ycao@vt.edu
} 
modeled directly in terms of elementary reactions (governed by the law of mass-action) ${ }^{25,26}$. A great advantage of this approach is that the newly designed deterministic model can be converted into its corresponding stochastic version without any approximation. A disadvantage of this approach is that we cannot re-purpose our original deterministic models, which had been carefully designed and parametrized to explain a broad scope of experimental observations. Furthermore, to model the phosphorylation and dephosphorylation reactions that play such important roles in cell-cycle progression introduces substantial complexity into the system. Recently we have considered a new approach that sidesteps the complications of elementary reactions and mass-action rate laws and that employs a Langevin-type simulation of noisy gene expression ${ }^{23}$. This approach, though promising, also requires an overhaul of the original deterministic models. In order to take advantage of existing deterministic models in a framework that permits accurate stochastic simulations without 'unpacking', we explore a particular hybrid approach in this paper.

Gillespie's SSA simulates every single reaction firing. In general, the time complexity of this algorithm scales proportionally with the number of reaction firings. Consequently, SSA-based models involve substantial computational complexity if a reaction network involves many fast reactions. To reduce the high computational cost of the SSA, many optimization methods ${ }^{39-43}$ and approximation methods ${ }^{44-48}$ have been proposed. Among them the hybrid stochastic approach, originally proposed by Haseltine and Rawling $(\mathrm{HR})^{46}$, performs well because it takes advantage of the multi-scale features common in biochemical reaction networks. The multiscale characteristics of reaction networks have led to significant reductions in the computational cost of solving many types of stochastic systems. For example, hybrid approaches provide good approximate solutions for the chemical master equation ${ }^{49,50}$.

The main idea of any hybrid approach is to divide the system into subsystems and solve each subsystem using an appropriate method. The idea of the HR hybrid approach (that we are using in this paper) is to partition the dynamical system into fast and slow reactions, based on the relative time scale of each reaction and the abundances of the reactants. Fast reactions, which fire frequently and often involve high-abundance species, are partitioned into the deterministic (ODE) regime. Meanwhile, slow reactions, which are often found at the gene-expression level, fire much less frequently and are therefore simulated using the SSA. This approach was first applied by Liu et al. ${ }^{22}$ on the simple three- variable model of the budding yeast cell cycle, originally studied by Kar et al. $^{20}$. By partitioning all gene-expression reactions into the slow (SSA) regime and all protein-level dynamics into the fast (ODE) regime, Liu et al. ${ }^{22}$ were able to reproduce the noise levels that Kar et al. ${ }^{20}$ achieved by unpacking the original system into a much more complex one. This success motivated us to apply this approach to the very comprehensive, accurate, and complex deterministic model of yest cell-cycle controls proposed by Chen et al. ${ }^{11}$.

Simulation results demonstrate that, while achieving high computational efficiency, our hybrid model still matches up well with experimental measurements of the variability of cell-cycle properties (cycle time, cell size, correlation coefficients), protein and mRNA abundances, and phenotypes of more than 100 mutant strains of budding yeast. Moreover, our simulations shed light on the 'partial' viability of mutant strains such as CLB2db $c l b 5 \Delta$.

\section{RESULTS}

We develop a hybrid stochastic model of the budding yeast cell cycle, consisting of 45 proteins and 19 mRNAs. In Section Methods, we will elaborate the steps for building our hybrid stochastic model. Building on this model, we use Algorithm 1, that we introduce in Section Methods to generate sufficiently large populations of mother and daughter cells to estimate the statistical distributions of various cell-cycle-related properties of wild-type cells as well as 122 mutant strains of yeast. We evaluate our model by comparing numerical simulation results with experimental observations from the published literature.

\section{Wild-type cell}

Figure $1 \mathrm{a}, \mathrm{b}$ shows a deterministic simulation of several protein and mRNA species, respectively, in our model of wild-type budding yeast cells. In early G1 phase, once the cell grows to a critical size, Cln3 and Bck2 initiate the START event, i.e., the activation of transcription factors for $\mathrm{Cln} 2$ and $\mathrm{Clb} 5$ production. $\mathrm{Cln} 2$ is the cyclin responsible for bud formation. In addition, Cln2 phosphorylates Sic1 and Cdc6, a pair of cyclin-dependent kinase inhibitors (CKIs). Consequently, Clb5-dependent kinase activity rises and initiates DNA replication in $\mathrm{S}$ phase. As CKIs are removed, $\mathrm{Clb} 2$ level rises, because $\mathrm{Clb} 2$ activates its own transcription factor, $\mathrm{Mcm} 1$, in an autocatalytic fashion. Clb2-dependent kinase activity
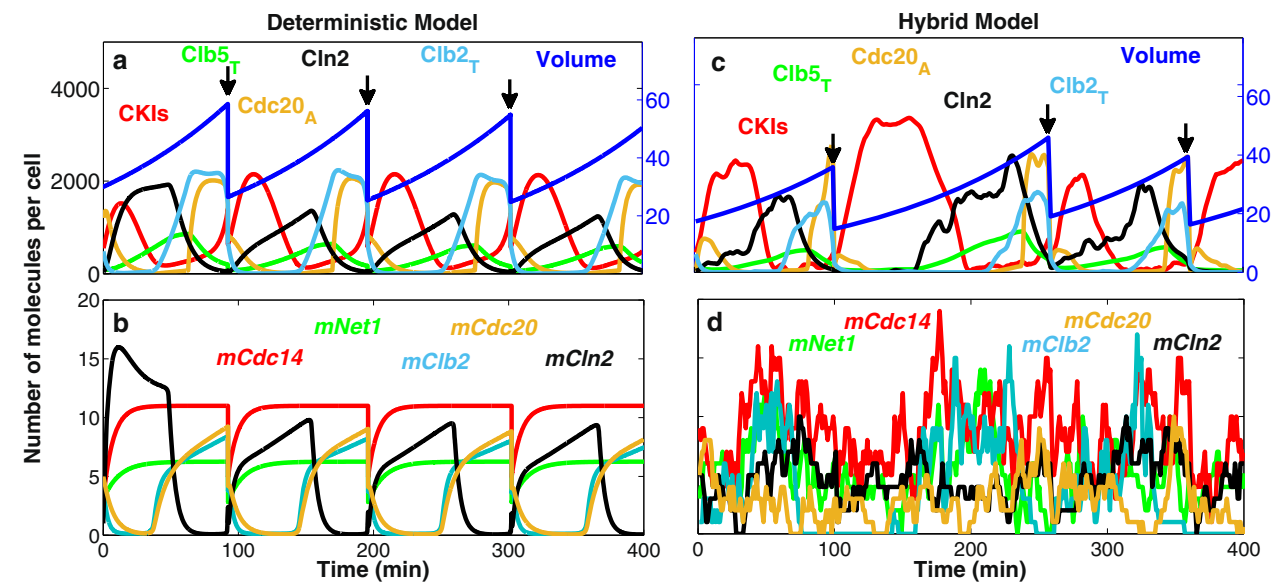

Fig. 1 Deterministic and hybrid stochastic simulations of the model. $\mathbf{a}, \mathbf{b}$ The temporal dynamics of representative proteins (a) and mRNAs (b) generated by the deterministic model. The volume of the cell increases exponentially and is divided (at the arrows) asymmetrically between mother $(55 \%)$ and daughter cell $(45 \%)$. c, d Stochastic simulation of the same proteins (c) and mRNAs (d) as in panels a and b, generated by a representative run of our hybrid stochastic model. Similar to the deterministic model, the cell grows exponentially; however, at the time of division all species in the cell, except for $C \ln 3$ and Bck2, are partitioned between daughter and mother cells with a 40:60 ratio, according to observations by Di Talia et al. ${ }^{28}$. Cln3 and Bck2, which are preferentially retained in mother cells ${ }^{56,57}$, are partitioned with a ratio 20:80 between daughter and mother cells. The daughter cell is tracked from division to division in this simulation. 
Table 1. Mean and coefficient of variation (CV) for cell-cycle properties.

\begin{tabular}{|c|c|c|c|c|c|}
\hline & & \multicolumn{2}{|l|}{ Mother cell } & \multicolumn{2}{|l|}{ Daughter cell } \\
\hline$T_{\mathrm{div}}(\min )$ & mean $\pm S E$ & $87 \pm 0.22$ & $87 \pm 1$ (116) & $111 \pm 0.36$ & $112 \pm 3(97)$ \\
\hline$T_{\mathrm{SG} 2 \mathrm{M}}(\min )$ & & $69 \pm 0.20$ & $72 \pm 1$ (116) & $73 \pm 0.26$ & $76 \pm 2(97)$ \\
\hline$V_{\text {birth }}(\mathrm{fL})$ & & $41 \pm 0.08$ & 40 & $27 \pm 0.05$ & 28 \\
\hline$T_{\mathrm{SG} 2 \mathrm{M}}(\mathrm{min})$ & & $0.30 \pm 0.0020$ & $0.17 \pm 0.02(116)$ & $0.36 \pm 0.0017$ & $0.2 \pm 0.06(97)$ \\
\hline$V_{\text {birth }}(\mathrm{fL})$ & & $0.28 \pm 0.0014$ & 0.18 & $0.28 \pm 0.0014$ & 0.20 \\
\hline
\end{tabular}

Mean \pm SE and CV \pm SE computed from simulation of the hybrid stochastic model are compared with experimental observations reported by Di Talia et al. ${ }^{28}$. The standard errors of the mean are in the same unit of the corresponding characteristic. The number of experimental observations are reported in parenthesis and the number of simulations used to calculate each quantity is at least $10,000 . T_{\text {div }}, T_{\mathrm{G} 1}, T_{\mathrm{SG} 2 \mathrm{M}}$, and $V_{\mathrm{birth}}$ are, respectively, cell-cycle duration or the time between two divisions, time from division to next emergence of bud, time from onset of bud to next division, and volume of the cell at birth.

turns off the transcription factors for $\mathrm{Cln} 2$ and $\mathrm{Clb5}$ production and is responsible for driving the cell into mitosis ( $\mathrm{M}$ phase). $\mathrm{Clb2}$ level remains high until metaphase, when the proper attachment of chromosomes to the mitotic spindle activates Cdc20. Cdc20 promotes anaphase (the separation of the two strands of replicated chromosomes to opposite poles of the mitotic spindle). At the same time, $\mathrm{Cdc} 20$ promotes degradation of $\mathrm{Clb} 2$ and $\mathrm{Clb} 5$ and activation of a phosphatase, $\mathrm{Cdc} 14$. Cdc14 plays a major role (in budding yeast) in re-establishing the dominance of CKIs in G1 phase, and in replacing $\mathrm{Cdc} 20$ by $\mathrm{Cdh} 1$ (the protein responsible for $\mathrm{Clb} 2$ and $\mathrm{Clb} 5$ degradation in G1 phase).

Figure 1c, d shows the corresponding stochastic trajectories of our hybrid stochastic model. The stochastic trajectories in panel $c$ correctly simulate the sequence of events predicted by the deterministic trajectories in panel a. Panel $d$ shows considerable fluctuations in the numbers of molecules per cell of five mRNA species: three of which $(m C l b 2, m C d c 20$, and $m C \ln 2)$ undergo periodic transcription during the cell cycle, and two of which ( $m C d c 14$ and $m N e t 1$ ) are synthesized continuously throughout the cell cycle. Such high variability is expected, due to the stochastic nature of gene expression and the low abundances of mRNA molecules per cell.

We used our hybrid stochastic model to generate more than 20,000 asynchronous wild-type mother and daughter cells growing in glucose medium (mass-doubling time about $100 \mathrm{~min}$ ). These large collections of simulated cells are then used to estimate the distributions of important characteristics of the budding yeast cell cycle, including inter-division time, duration of unbudded phase (G1), duration of budded phase (S-G2-M), and size at birth. In addition we estimate the standard error (SE) of mean and standard error of coefficient of variation for these characteristics. Table 1 compares the computed summary statistics \pm SE for all cell-cycle-related properties with experimental data reported by Di Talia et al. ${ }^{28}$. The results in Table 1 show that the model accurately reproduces the mean of these important properties of the wild-type budding yeast cell cycle. Despite the fact that the coefficients of variation reproduced by our model are generally larger than what is observed in experiment, they are in a comparable range. In accord with experimental observations, G1 phase is the noisiest phase in cell cycle, the variability in daughter cells is more than mother cells. The estimated standard errors are significantly smaller than the experimental observations. In fact, we expect such low standard errors due to the large number of simulations. We note that the standard error for volume of a cell at birth is not reported in column 4 and 6 , because cell volume is not measured directly by $\mathrm{Di}$ Talia et al. ${ }^{28}$, but rather $V(t)$ is estimated as a function of time.

Next, we compare our simulations to the observed distributions of mRNA molecules in wild-type yeast cells. We have 11 unregulated mRNAs ( $m C d h 1$, mTem1, mCdc15, mCdc14, mNet1, $m(d c 55, m E s p 1, m S B F, m M B F, m M c m 1, m A P C)$ and eight transcriptionally regulated mRNAs ( $m C l b 5, m C l n 2, m C l b 2, m S i c 1$, $m C d c 6, m S w i 5, m C d c 20, m P d s 1$ ) in our model. Figure 2 compares the histograms of these mRNAs with the distributions measured by Ball et al. ${ }^{27}$. In the original deterministic model, MBF and SBF are described by the same algebraic equation since they were supposed to have the same dynamics ${ }^{11}$. In adding $m S B F$ and $M M B F$ to the model, we kept the same assumption and therefore, the histograms of the two unregulated mRNAs ( $m S B F$ and $m M B F$ ) are very similar. For this reason we just include one of them $(m S B F)$ in Fig. 2. To quantify the difference between a distribution generated by our model and the corresponding experimental observations, we use the Kullback-Leibler divergence $\left(D_{\mathrm{KL}}=\sum_{x \in X} R(x) \log \left(\frac{Q(x)}{R(x)}\right)\right)$ where $R$ is the distribution from simulation and $Q$ from experiment. The computed value of the $\mathrm{KL}$ divergence is reported on the top-left corner of each subplot. The smaller is $D_{\mathrm{KL}}$, the more similar are the two distributions.

Generally, there is a good agreement between observed and simulated mRNA distributions, except for $m C \ln 1+m C \ln 2$ and $m C d c 20$. As expected, the unregulated transcripts follow Poisson distributions, which are consistent with experimental measurements. The value of $D_{\mathrm{KL}}$ computed for these distribution is small. The cellcycle regulated transcripts, which follow long-tailed, non-Poisson distributions, are well-fit by two-component Poisson distributions as reported by refs ${ }^{26,27}$. (We note that in our model $\mathrm{mClb} 2$ represents both $m C l b 1$ and $m C l b 2$, and $m C \ln 2=m C \ln 1+m C \ln 2$, whereas in the experiment these cyclin mRNAs are tracked independently. Therefore, we do not expect a particularly good match between the computed and observed distributions for these transcripts. As expected, the values of $D_{\mathrm{KL}}$ computed for these distribution are large).

Table 2 compares the average abundances of proteins as observed in ref. ${ }^{51}$ and simulated by our model. We use a sufficiently large population of cells from at least 10,000 simulations to calculate the average abundance (number of molecules per cell) and the standard error of the mean for each protein. Note that, for the proteins listed in Table 2, only a single measurement has been made experimentally, so the standard error is not 


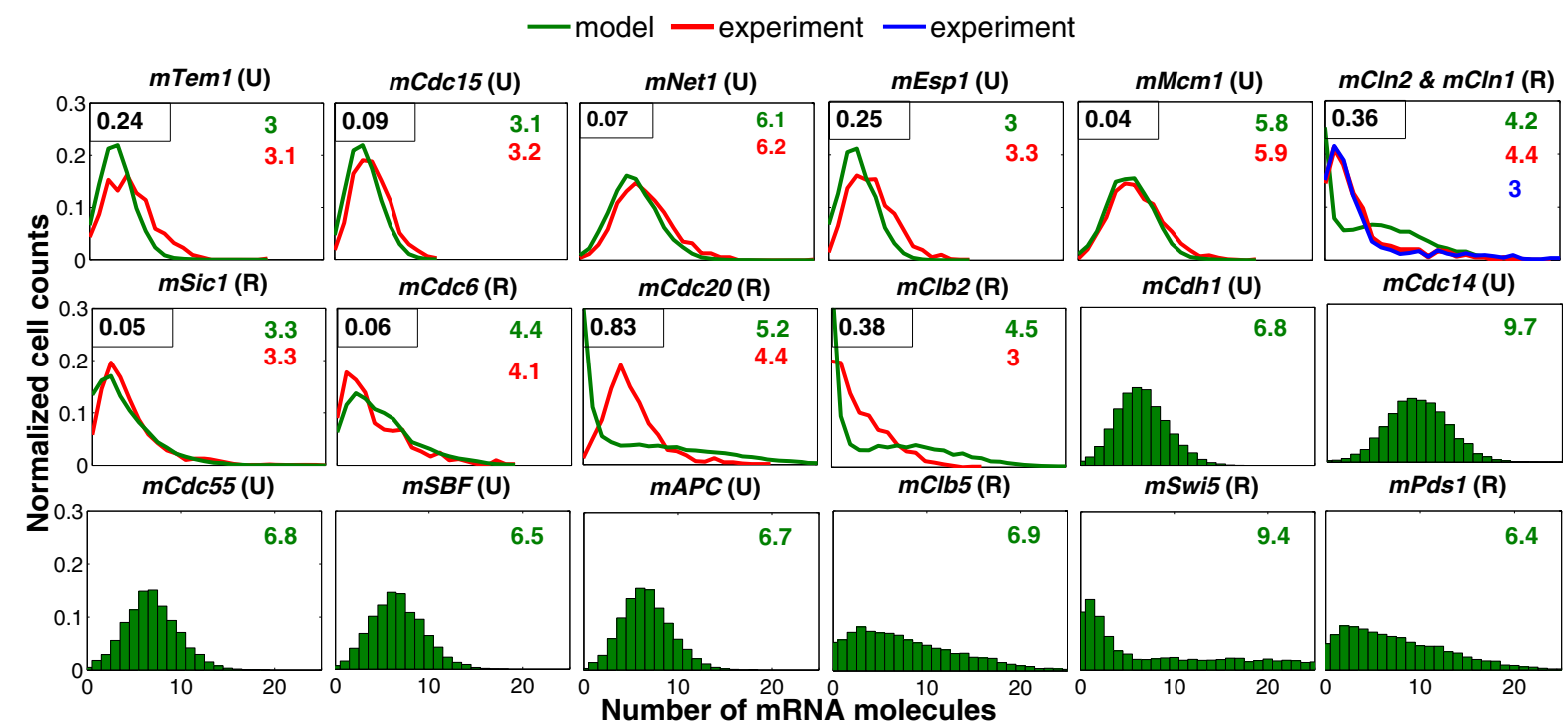

Fig. 2 Histograms of mRNAs for a population of wild-type cells growing in glucose medium. The histograms of mRNA molecules generated from a stochastic run of the hybrid model (in green) are compared with experimental observations ${ }^{27}$ (in red and blue colors) for a population of wild-type cells growing in glucose. (In the simulation the growth rate is set to $0.0072 \mathrm{~min}^{-1}$ to reproduce the 96 min mass-doubling time of wild-type cells growing in glucose culture medium.) $U$ and $R$ in parenthesis indicate, respectively, unregulated and transcriptionally regulated mRNAs. The histograms in red are reproduced from the experimental data reported by Ball et al. ${ }^{27}$. For the last eight transcripts, experimental data are not available. On the top-right corner the average number of mRNA molecules is compared with experiment where available. On the top-left corner the Kullback-Leibler divergence $\left(D_{K L}\right)$ is reported to quantify the difference between the two distributions. $D_{K L}=0$ indicates that the two distributions in question are identical. In our model $m C \ln 2$ stands for $m C \ln 1+m C \ln 2$. In experiment, however, they are measured separately. Here, the histograms in red and blue are, respectively, $m C \ln 1$ and $m C \ln 2$. Similarly, in our model $m C l b 2$ describes the abundance of both $m C l b 1$ and $m C l b 2$; however, the histogram reproduced from the experimental data refers only to $m C l b 2$.

Table 2. Average abundances of protein molecules per cell.

\begin{tabular}{|c|c|c|c|c|c|}
\hline Protein & \multicolumn{2}{|c|}{ Average abundance \pm standard error } & Protein & \multicolumn{2}{|c|}{ Average abundance \pm standard error } \\
\hline$C \ln 3$ & 108 & $109 \pm 2$ & $\mathrm{Swi5}_{T}$ & 688 & $658 \pm 6$ \\
\hline $\mathrm{Cln} 2_{T}=\mathrm{Cln} 1+\mathrm{Cln} 2$ & $1589=319+1270$ & $1647 \pm 18$ & Tem1 & 573 & $544 \pm 11$ \\
\hline $\mathrm{Clb}_{T}$ & 420 & $516 \pm 5$ & Cdc15 & 238 & $257 \pm 4$ \\
\hline $\mathrm{Clb}_{T}$ & 693 & $736 \pm 12$ & $\operatorname{Net} 1_{T}$ & 1590 & $1579 \pm 8$ \\
\hline
\end{tabular}

The average abundance \pm standard error of proteins in molecules per cell, computed by the hybrid stochastic model, are compared with experimental observations reported in ref. ${ }^{51}$. In our model $\mathrm{Clb} 5$ stands for $\mathrm{Clb} 5$ and $\mathrm{Clb} 6, \mathrm{Clb} 2$ stands for $\mathrm{Clb} 1$ and $\mathrm{Clb} 2$, and $\mathrm{Cln} 2$ stands for $\mathrm{Cln} 1$ and $\mathrm{Cln} 2$. We are reporting the total abundance of each protein, which includes protein molecules that are either phosphorylated or unphosphorylated, and that are bound in complexes or free. That is, $\mathrm{Clb}_{\mathrm{T}}=\mathrm{Clb} 5+\mathrm{C} 5+\mathrm{C} 5 \mathrm{P}+\mathrm{F} 5+\mathrm{F} 5 \mathrm{P}, \mathrm{Clb} 2_{\mathrm{T}}=\mathrm{Clb} 2+\mathrm{C} 2+\mathrm{C} 2 \mathrm{P}+\mathrm{F} 2+\mathrm{F} 2 \mathrm{P}$, and $\mathrm{Sic} 1_{\mathrm{T}}=\mathrm{Sic} 1+\mathrm{Sic} 1 \mathrm{P}+\mathrm{C} 2+\mathrm{C} 2 \mathrm{P}+\mathrm{C} 5+\mathrm{C} 5 \mathrm{P}$.

available for comparison. Except for the Sic1, the agreement between simulation results and experimental observations is quite reasonable.

\section{Mutant cases}

Our hybrid stochastic model is used to simulate 122 mutant strains listed in Table S5 in Supplementary Text. Prior to presenting simulation results of these mutants, we discuss the criteria for determining viability in a general stochastic model.

In the original deterministic model, a cell is considered viable if the following conditions are met:

(1) certain events, listed in ref. ${ }^{35}$, take place in a proper sequence,

(2) in particular, cell division occurs after budding, and

(3) cell mass does not exceed a predetermined threshold (mass at division $<10$ ).
The same set of rules should also apply to the stochastic model. A division is considered successful if the aforementioned viability criteria are met; otherwise, the cell is considered to be inviable. From our numerical simulations, the probability of successful division $p$ is estimated by

\section{Number of successful divisions \\ $p=\overline{\text { Number of successful divisions }+ \text { number of failures }}$.}

Using this metric, we consider a mutant strain viable if $p>0.75$, inviable if $p<0.65$, and partially viable otherwise. This viability criterion is based on the following considerations.

Let $N_{0}$ be the initial population of cells in an experiment. After one cycle, the average number of cells that divide is $p N_{0}$, while $(1-p) N_{0}$ cells exit the cycle and stop dividing. Thus, after one cycle, the total population of cells is $(1+p) N_{0}$, of which $2 p N_{0}$ cells completed the previous cell cycle and $(1-p) N_{0}$ cells have ceased 
to divide (we call them dead cells). In our simulations, we disregard the $(1-p) N_{0}$ dead cells; hence, the number of actively dividing cells in the second cycle is $N_{1}=2 p N_{0}$, and the expected number of actively dividing cells after $k$ cycles will be $N_{k}=(2 p)^{k} N_{0}$.

In cell-viability experiments, colony formation is typically assessed after $24 \mathrm{~h}$ growth of a series of ten-fold diluted inocula. For wild-type yeast cells $(p \approx 1)$ growing on rich glucose medium (cycle time $\approx 12 \mathrm{~h}$ ), each inoculum should increase by a factor of about $2^{12}=4096$. The colony sizes after $24 \mathrm{~h}$ growth of ten-fold serial dilutions would be $\left(4000 N_{0}, 400 N_{0}, 40 N_{0}, 4 N_{0}\right)$, of which the first would be too dense to quantify, the last would be too sparse to see, and the middle two would be used to assess viability of mutant strains. For a mutant cell with $p<0.5$, no visible colony will grow from the initial inoculum, and the mutant will be scored 'inviable'. For mutant strains with $0.5<1$, we must consider how the colony growth assay compares to wild-type cells. For $p=$ 0.8 the initial inoculum grows to $280 N_{0}$, which is comparable to the first dilution of the wild-type cells, and we would score this mutant strain as 'viable'. For $p=0.75$ the initial inoculum grows to $130 N_{0}$, which is denser than the second dilution of the wild-type cells, and we would score this mutant strain as 'probably viable'. For $p=0.65$ the initial inoculum grows to $23 N_{0}$, which is less dense than the second dilution of the wild-type cells, and we would score this mutant strain as 'hardly viable'. These calculations suggest that a mutant strain be considered viable if $p \geq 0.75$ and nonviable if $p \leq 0.65$. For $0.65<p<0.75$, the strain is identified as partially viable.

Based on these criteria, we assessed the viability of 122 mutant strains of budding yeast that were studied in the modeling paper of Chen et al. ${ }^{11}$. To demonstrate the significant roles of noise in some of these mutants, we discuss two multiple-mutant strains, $c \ln 1 \Delta c \ln 2 \Delta b c k 2 \Delta$ and $c \ln 3 \Delta b c k 2 \Delta$ multi-copy $C L N 2$ (Fig. 3), in some detail. Our goal is to illustrate how we assess the viability of a mutant strain in our hybrid stochastic model. According to experimental observations, the $c \ln 1 \Delta c \ln 2 \Delta b c k 2 \Delta \operatorname{strain}^{52}$ is viable. However, due to deletion of START cyclins Cln1 and Cln2, the cell requires a longer time than normal to form a bud and hence grows to a larger size at division, in comparison with wildtype cells. Figure 3a shows that in the deterministic model the cell consistently exits mitosis and divides successfully with size larger than normal, as observed experimentally ${ }^{52}$. In the hybrid stochastic simulation, however, due to the stochastic nature of the process there is a finite probability that a cell may exit the cycle and become arrested in some phase of the cell cycle. In Fig. $3 \mathrm{~b}$ for instance the cell grows too large in G1 phase and never divides again, while in Fig. $3 c$, it exits mitosis and divides successfully. The probability of successful completion of the cell division cycle, in this case, is computed to be $p \approx 0.84$. As shown in Fig. $3 d$, the total number of cells in our computational culture increases exponentially, with a number-doubling time (NDT) of 140 min, which is slower than the NDT of a fully viable wild-type culture $(\sim 100 \mathrm{~min})$. Therefore, we conclude that the hybrid stochastic simulation correctly confirms the viability, but the reduced growth rate, of the $c \ln 1 \Delta c \ln 2 \Delta b c k 2 \Delta$ strain.

Next we consider the inviable mutant strain $\ln 3 \Delta b c k 2 \Delta$ multicopy $C L N 2^{53}$. Figure $3 e$ shows that in the deterministic model the mutant cell is arrested in G1 phase and grows without dividing until it dies. In the hybrid stochastic simulation, although many of the cells become arrested in G1 (see Fig. 3f), some cells manage to exit G1 phase, complete the cell cycle, commence a new cycle and divide a few times (see Fig. $3 \mathrm{~g}$ ). Nonetheless, according to Fig. $3 \mathrm{~h}$ the total number of cells in our computational culture declines with time, because the probability of cell division is only $p \approx 0.40$. Therefore, we conclude that the hybrid stochastic simulation correctly confirms the inviability of $\ln 3 \Delta b c k 2 \Delta$ multi-copy CLN2 strain.

Based on our hybrid stochastic simulations of all 122 mutant strains in Chen's data base, we find that the model successfully reproduces the phenotypes of 103 of these strains. Our results for all mutant strains are reported in Table S5 in Supplementary Text.

$C L B 2 d b \triangle c l b 5 \Delta$ is a mutant with an interesting stochastic phenotype: it is inviable when grown on glucose medium but 'partially viable' when grown on raffinose (a sugar that supports a slower growth rate than glucose) ${ }^{54}$. Due to deletion of the destruction box of CLB2, Clb2 protein is in excess at telophase and the cell is unable to exit mitosis and divide, even in the absence of Clb5 protein (due to deletion of the CLB5 gene). When growing on raffinose, however, many of these mutant cells (approximately $60 \%-75 \%$ ) are able to exit mitosis and commence a new cycle, whereas the remaining cells $(25 \%-40 \%)$ are arrested in telophase and never re-enter the cell cycle ${ }^{21}$. In fact, the NDT of the doublemutant cells $(250-300 \mathrm{~min}$ ) is observed to be much longer than the NDT of wild-type cells $(160 \mathrm{~min})$ growing in raffinose medium $^{21}$.

Simulation results of Chen's deterministic model predict that $C L B 2 d b \Delta c l b 5 \Delta$ cells are inviable on glucose and viable on galactose and raffinose media. Clearly, we cannot expect a deterministic model to capture the stochastic properties of such a 'partially viable' mutant strain. Our hybrid stochastic model, however, describes the phenotype in exquisite detail. The probability of division for $C L B 2 d b \Delta c l b 5 \Delta$ strain is $p \approx 0.68$ confirming the partial viability of the mutant according to our viability criterion. Figure $4 \mathrm{a}$ shows the cumulative probability, $P(T)$, of cycle times for wild-type and CLB2db $\Delta$ clb5 $\Delta$ mutant cells growing in raffinose. $(P(T)$ is the probability that the cycle time of a randomly chosen cell is longer than a specified time, $T$.) As shown in Fig. 4a, $P(T)$ for the mutant cells levels off at approximately $35 \%$ as $T$ increases, whereas, for wild-type cells, $P(T)$ drops steadily (below 5\%) as $T$ increases beyond $250 \mathrm{~min}$. Cumulative distributions of cycle times computed by our hybrid stochastic model (black lines) are in excellent agreement with the experimental distributions (red-blue-green lines) for both wildtype and the double-mutant cells.

Another interesting mutant strain is $C L B 1 c l b 2 \Delta c d h 1 \Delta$, for which the $C L B 1$ gene is intact and CLB2 and $C D H 1$ genes are deleted. Due to the mutual antagonism between $\mathrm{Clb} 2$ and $\mathrm{Cdh} 1$, deletion of both genes, $C D H 1$ and $C L B 2$, might be consistent with viability of the double-mutant strain, provided $\mathrm{Clb} 1$ is still functional. Indeed, experimental observations show that $C L B 1 c l b 2 \Delta c d h 1 \Delta$ cells are poorly viable in glucose medium and viable when growing on galactose ${ }^{54}$. Chen's deterministic model does not capture this phenotype; the model predicts the mutant cells to be viable in both media. However, simulation results of our hybrid stochastic model (Fig. 4b) can reproduce the observed phenotype. The probability of division computed for a population of cells growing in glucose is $\approx 0.62$, which suggests poor viability. In galactose the corresponding probability of division is $\approx 0.79$, which indicates that the mutant grows well in this medium. Figure $4 \mathrm{~b}$ confirms a faster increase in cell number in the slower growth medium (galactose), which is in agreement with experimental observations.

\section{DISCUSSION}

In this paper we present a hybrid stochastic model of the molecular mechanism controlling progression through the budding yeast cell cycle. Our model provides a good match with experimental observations of many important characteristics of the budding yeast cell cycle, including inter-division time, cell size, and the phenotypes of more than 100 mutant strains. Compared with other approaches to stochastic modeling, our hybrid stochastic approach has several advantages. In a multi-scale regulatory network such as cell-cycle controls, the major source of intrinsic noise can be attributed to low copy numbers of mRNA species in the gene-protein regulatory network. In fact, in budding yeast cells there are only 5-10 copies of each mRNA species 

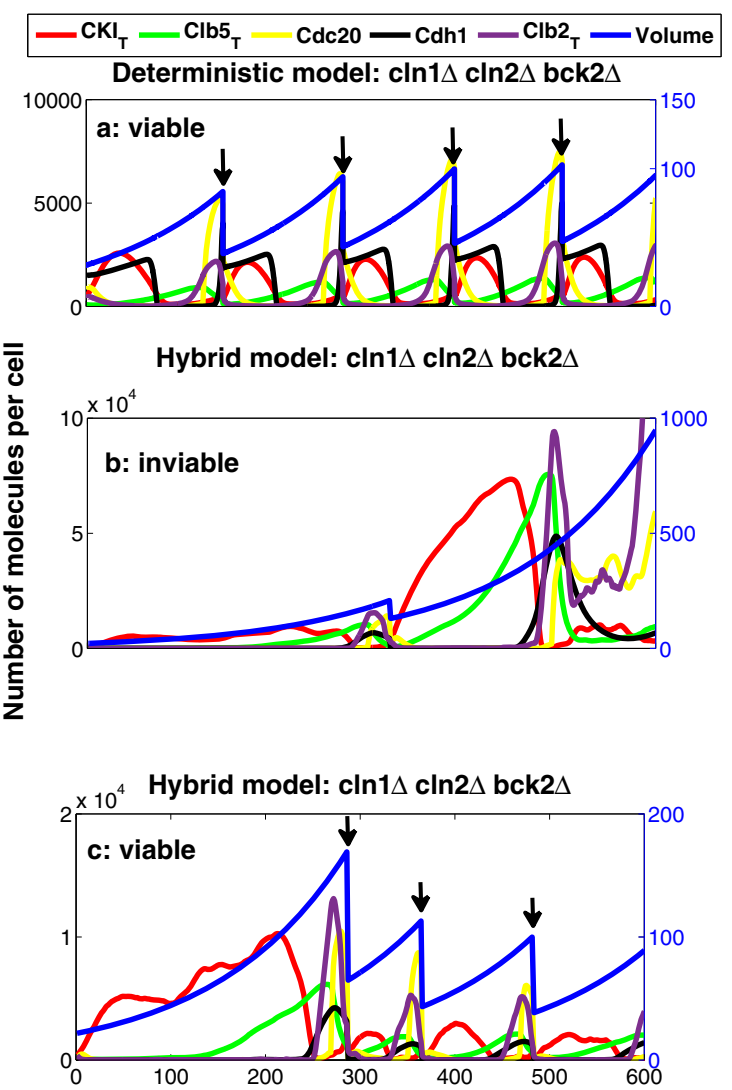

Hybrid model: $\operatorname{cln} 1 \Delta \operatorname{cln} 2 \Delta$ bck2 $\Delta$

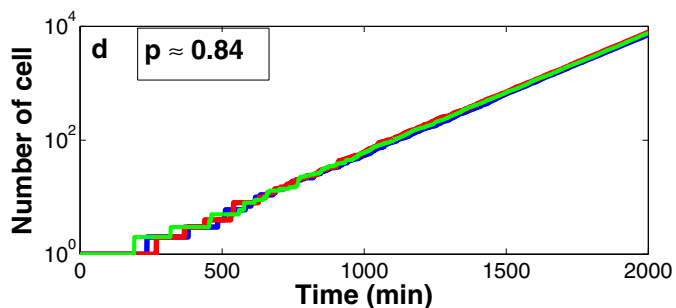

Deterministic model: cln3 $\Delta$ bck2 $\Delta$ multi-copy CLN2

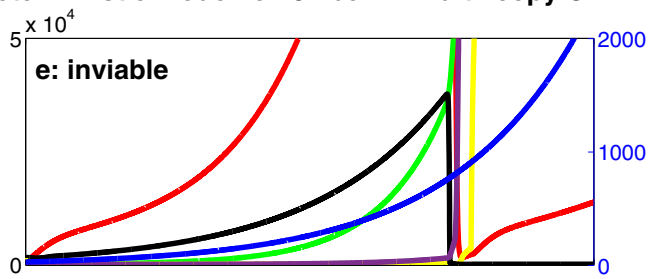

Hybrid model: $\operatorname{cln} 3 \Delta$ bck2 $\Delta$ multi-copy CLN2

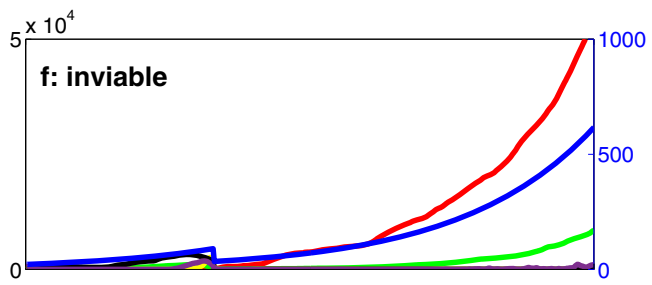

Hybrid model: cln3 $\Delta$ bck2 $\Delta$ multi-copy CLN2

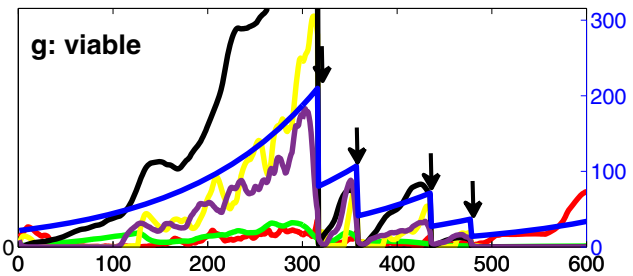

Hybrid model: cln3 $\Delta$ bck2 $\Delta$ multi-copy CLN2

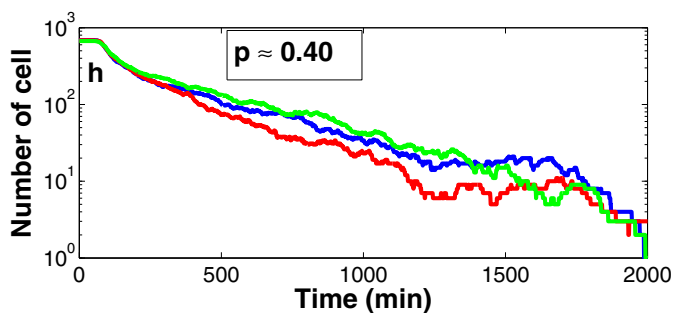

Fig. 3 Comparison of deterministic and stochastic trajectories of two different multiple-mutant strains. a Deterministic trajectories of $c \ln 1 \Delta \ln 2 \Delta b c k 2 \Delta$; the cell consistently exits mitosis and divides (the divisions are indicated by arrows). b, c Stochastic trajectories of $c \ln 1 \Delta \ln 2 \Delta b c k 2 \Delta$ from two independent runs. In panel b the cell becomes arrested in $\mathrm{G} 1$ phase while in panel c the cell divides successfully. d The total number of cells as a function of time; we start each simulation with one cell and count the total number of cells over time for $2000 \mathrm{~min}$. The probability of division is calculated as $p \approx 0.84$ which indicates that the $\ln 1 \Delta c \ln 2 \Delta b c k 2 \Delta$ strain is viable according to our definition. The semilog plot in panel $\mathbf{d}$ shows that the number of cells increases exponentially (NDT $\approx 140$ min) in our computational culture. e Deterministic trajectories of $c \ln 3 \Delta b c k 2 \Delta$ multi-copy $C L N 2$; the cell arrests permanently in G1 phase. f, g Stochastic trajectories of $c l n 3 \Delta b c k 2 \Delta$ multi-copy CLN2 from two independent runs. In panel $\mathbf{f}$ the cell becomes arrested in G1 phase after one cycle, while in panel $\mathbf{g}$ the cell exits mitosis and divides successfully several times. $\mathbf{h}$ The total number of cells as a function of time; we start the simulation with 1000 cells and count the total number of viable cells over time for $2000 \mathrm{~min}$. The probability of division is calculated as $p \approx 0.40$ which indicates that the $c \ln 3 \Delta b c k 2 \Delta$ multi-copy CLN2 strain is inviable. The semilog plot in panel $\mathrm{h}$ shows that the total number of cells decreases exponentially in our computational culture.

encoding the production of corresponding proteins at levels of 500-5000 molecules per cell. In such circumstances, small fluctuations in the population of mRNAs will result in substantial fluctuations in the corresponding protein levels. With this in mind, the key idea of the hybrid scheme is to partition the dynamics of mRNA species into the stochastic regime, in order to capture the major effects of random fluctuations in mRNA numbers, and to keep the protein dynamics in the deterministic framework, to achieve greater simulation efficiency. In addition, in this scheme it is not necessary to reformulate the complex rate laws governing protein interactions as elementary mass-action rate laws, which is a great advantage from a modeling standpoint.
In this paper, we have applied our hybrid stochastic method to a detailed molecular mechanism of cell-cycle controls in budding yeast ${ }^{11}$. To apply our scheme to Chen's model, which is a deterministic model of protein interactions, we first had to extend the model to include mRNA species that are transcribed from cellcycle genes and translated into proteins. Then we carried out comprehensive simulations of wild-type yeast cells and more than 100 mutant strains, using both the deterministic and hybrid ODE/ SSA models. Our stochastic model predicts the statistical properties of many different cell-cycle variables, including inter-division times, size at birth, and the abundances of specific mRNAs and proteins, and our stochastic simulations are in accord with most experimental observations, including detailed phenotypic 

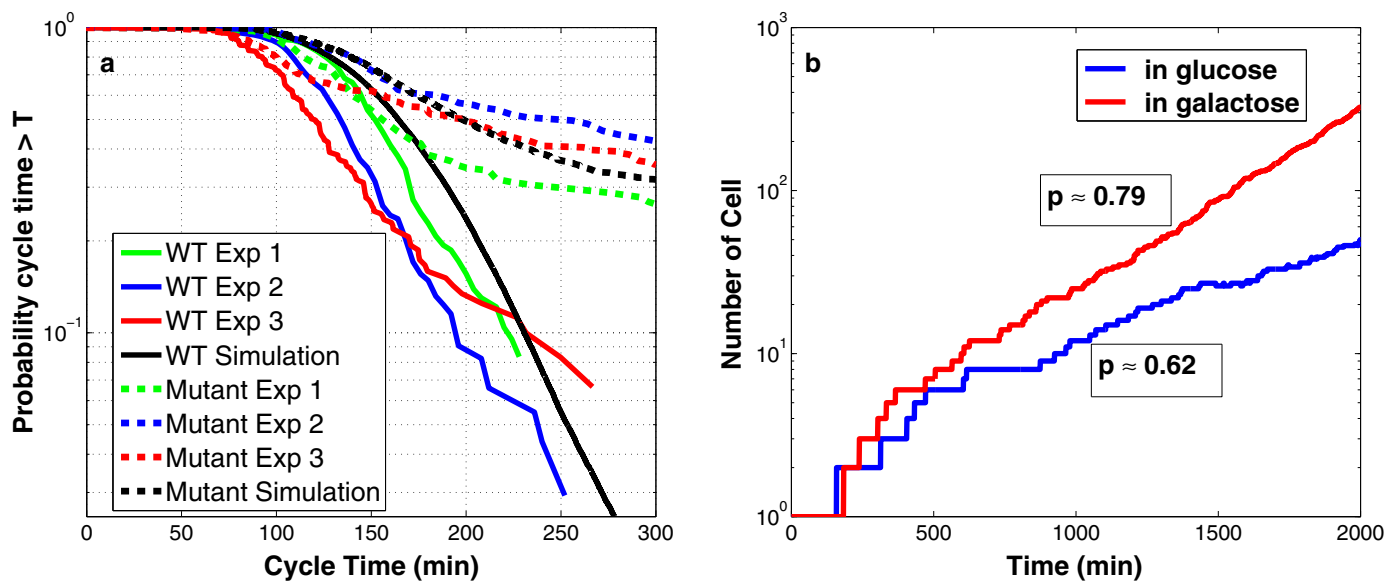

Fig. 4 Stochastic phenotypes of two more mutant strains. a Comparison of wild-type and $C L B 2 d b \Delta$ clb5 $\Delta$ mutant cells growing in raffinose. The probability that a cell divides with a cycle time longer than a specific time $T$ is plotted for wild-type cells (solid lines) and mutant cells (dotted lines). The black lines are generated by our hybrid stochastic model and the red-blue-green lines are the results of three independent experimental runs by Ball et al. ${ }^{21}$. To model growth on raffinose medium in our simulation, the specific growth rate of cells is set to 0.00433 $\min ^{-1}$ (MDT $=160 \mathrm{~min}$ ). b Comparison of cell proliferation for colonies of CLB1 clb2 $2 \mathrm{cdh} 1 \Delta$ cells growing in glucose (blue) or galactose (red). The probability of division in our computational culture is given in the boxes next to each simulation. To mimic growth in glucose and galactose media, respectively, the specific growth rates are set to 0.0072 and $0.004621 \mathrm{~min}^{-1}$, i.e., MDT $=96$ and 150 min, respectively.

characteristics of 103 out of 122 mutant cases. (Although Chen's deterministic model may seem to 'score' better on the 'viability' of mutant strains, it is not so highly constrained as our stochastic model by consideration of the statistical properties of these mutant cells, especially the characteristics of 'partially viable' mutants.) In addition, our results prove that our hybrid approach to stochastic/deterministic simulations can achieve a good tradeoff between accuracy and efficiency of numerical simulations. FORTRAN code takes about $15 \mathrm{~min}$ to simulate 10,000 cell cycles on an Intel i7-3770 processor with $16 \mathrm{G}$ memory running a Linux environment. A similar system using a fully stochastic model may take more than one day (for example, when the FORTRAN code of Barik et al. ${ }^{26}$ is run using the same work station, it takes more than $30 \mathrm{~h}$ to generate a population of 10,000 yeast cells).

\section{METHOD}

Deterministic model

A comprehensive continuous-deterministic model of the budding yeast cell cycle was developed by Chen et al. $^{11}$ in 2004 . By integrating the findings of decades of experimental studies, Chen's model provides an accurate mathematical description of the cell division cycle of budding yeast. The protein regulatory network of Chen's model focuses primarily on the mutual antagonism between mitotic B-type cyclins (Clb1-6) and G1 phase stabilizers (Cdh1, Sic1, and Cdc6). During the growth and division of yeast cells, this antagonism leads to transitions between two coexisting steady states called StART $(\mathrm{G} 1 \rightarrow \mathrm{S})$ and $\operatorname{ExIT}(\mathrm{M} \rightarrow \mathrm{G} 1)$. A detailed description of the budding yeast cell-cycle model is given in Supplementary Information.

Chen's mathematical model reproduces the average cell-cycle properties (including cycle time, G1 duration, and cell size at division) of wild-type budding yeast cells and the variant cell-cycle phenotypes of more than 100 mutant strains. Our goal is to develop a hybrid (stochastic-deterministic) version of this large regulatory network, in order to quantify the variabilities observed in cell-cycle characteristics and mutant phenotypes within a computationally efficient framework.

Since Chen's model is formulated in terms of normalized (dimensionless) concentrations of proteins, the first step to this goal is to convert the state variables of Chen's model into integer numbers of molecules per cell. This conversion facilitates comparison of our numerical simulation results with observed data from single-cell experiments. Furthermore, it is necessary because, in Gillespie's SSA, state variables are discrete (species populations) rather than continuous (species concentrations). Since a hybrid model involves both SSA and ODEs, it is important that we assure consistency between units of state variables in both the stochastic and deterministic regimes. Therefore, we calculate $S_{i}$, the number of molecules of species $i$ in a cell, from the corresponding normalized concentration, $\left[S_{i}\right]_{n}$, by $(1)$ :

$S_{i}(t)=N_{\mathrm{A}} \cdot\left[\mathrm{S}_{i}\right](t) \cdot V(t)=0.6 \cdot C_{i} \cdot\left[\mathrm{S}_{i}\right]_{\mathrm{n}}(t) \cdot V(t)$,

where $\left[S_{i}\right]$ is the actual concentration of species $i$ (in nanomoles/liter $=$ $10^{-9} \mathrm{~mol} / \mathrm{L}$ ), $C_{i}$ is the 'characteristic' concentration of species $i$ (used to convert between actual concentration and 'normalized' concentration), $V(t)$ is the volume of the cell (in femtoliters $=10^{-15} \mathrm{~L}$ ), and $N_{\mathrm{A}}=0.6$ is Avogadro's number (when concentration is expressed in $\mathrm{nM}$ and volume in $\mathrm{fL}$ ). One simplifying assumption made in published models ${ }^{21,23}$ is to use a constant volume for the size of cell. However, this unrealistic assumption introduces errors into the model because cell size $(V)$ increases exponentially during a cycle $\left(V(t)=V(0) \mathrm{e}^{k_{g} t}\right.$, where $k_{g}$ is the specific growth rate of yeast cells).

Second, we extend the protein regulatory network in Chen's model to include the dynamics of 11 regulated and eight unregulated mRNAs. This extension is necessary because the major source of intrinsic noise in yeast cells is the small number of mRNA molecules per cell per gene ${ }^{27}$. Experimental observations ${ }^{28}$ in yeast cells with increased dosage of genes suggest that the dominant source of variability with respect to cell-cycle time and cell size at division is the low copy number of mRNA and protein molecules in a cell, specifically in G1 phase. However, Chen's model did not incorporate the turnover of mRNA molecules, and thus it cannot account for fluctuations stemming from transcriptional noise. For these reasons, Chen's original model must be supplemented with appropriate synthesis and degradation rates for each mRNA, as well as realistic rates of translation from mRNA to protein. In our model, based on experimental observations $\mathrm{in}^{55}$, we assigned half-life times for mRNAs in the range of 5-10 min, except for $m C \ln 2$ and $m C l b 2$, which were assigned shorter halflives ( 3 and 2 min, respectively). The synthesis rate of each mRNA was then estimated to match the mRNA average-abundance measurements in Ball et al. ${ }^{27}$.

Third, we modified Chen's model by introducing ODEs for the concentrations of $\mathrm{Cln} 3$ and Bck2 proteins. In Chen's original model, the normalized concentration of $\mathrm{Cln} 3$ and $\mathrm{Bck} 2$ were assumed to be given by steady-state algebraic equations (2) and (3),

$[\mathrm{Cln} 3]_{n}=\frac{C_{0} \cdot D_{\mathrm{n} 3} \cdot \text { mass }}{J_{\mathrm{n} 3}+D_{\mathrm{n} 3} \cdot \text { mass }}$

$[\mathrm{Bck} 2]_{n}=B_{0} \cdot$ mass,

where $C_{0}$ determines the maximum concentration of $\operatorname{Cln} 3, D_{\mathrm{n} 3}$ is the dosage of the CLN3 gene, $J_{\mathrm{n} 3}$ and $B_{0}$ are constants, and mass is the 'size' of a cell. We replaced the algebraic equations (2) and (3) by ODEs 
in (4) and (5).

$\frac{\mathrm{d} C \ln 3}{\mathrm{~d} t}=k_{\mathrm{s}, \mathrm{n} 3} \cdot V^{2}(t)-k_{\mathrm{d}, \mathrm{n} 3} \cdot C \ln 3$,

$\frac{\mathrm{d} B c k 2}{\mathrm{~d} t}=k_{\mathrm{s}, \mathrm{k} 2} \cdot V^{2}(t)-k_{\mathrm{d}, \mathrm{k} 2} \cdot B c k 2$

The synthesis $\left(k_{\mathrm{s}, \mathrm{n} 3}, k_{\mathrm{s}, \mathrm{k} 2}\right)$ and degradation $\left(k_{\mathrm{d}, \mathrm{n} 3}, k_{\mathrm{d}, \mathrm{k} 2}\right)$ rate constants were estimated so that the half-lives and average abundances of these proteins match with experimental data ${ }^{51}$. The reason for this change is to model the unbalanced partitioning of $\mathrm{Cln} 3$ molecules between daughter and mother cells at cell division. According to experimental observations, the concentration of $\mathrm{Cln} 3$ in a new-born daughter cell is about three times less than its concentration in the mother cell ${ }^{56,57}$, indicating that mother cells get more than their 'fair share' of Cln3 molecules at cell separation. As a consequence of this unequal partitioning of $\mathrm{Cln} 3$ between mother and daughter cells at division, the G1 time of mother cells is much shorter and the G1 time of daughter cells is much longer (on average) than would otherwise be expected. By including Cln3 and Bck2 as state variables in the model, we can apply an asymmetric partitioning rule with ratio of 20:80 to daughter and mother cells at cell division. We note that this ratio is set to 40:60 for all other proteins and mRNAs, according to observations in ${ }^{28}$.

Finally, we comment that the quadratic dependence of $\mathrm{Cln} 3$ and Bck2 synthesis rates on cell size is introduced to account for the major influence that these two proteins have on cell size at the G1/S transition $^{58,59}$. Because the rate of synthesis of these two proteins increases quadratically with cell volume, there is a strong size control on the G1/S transition in our model.

In summary, the variables, equations, parameter values and reaction propensities in our model are provided in Tables S1-S4 in Supplementary Text.

\section{Hybrid stochastic model}

As we mentioned in Section 1, the regulatory network of the budding yeast cell-cycle is a multi-scale system: both the numbers of molecules of mRNAs and proteins and the propensities of individual reactions vary by orders of magnitude. For instance, in budding yeast cells, there are 500-5000 copies of each protein encoded by only 5-10 copies of the corresponding mRNA. Furthermore, the synthesis and degradation of mRNA species occur much less frequently than the phosphorylation and dephosphorylation of proteins in the cell. The Haseltine and Rawling (HR) hybrid method leverages these large scale differences to improve the efficiency of stochastic simulations without sacrificing accuracy of the computations. The HR method divides the system into subsystems, each including species and reactions with similar scales, and applies an appropriate simulation method to each subsystem. This partitioning is done by using predefined thresholds for propensities of reactions and abundances of reactants. In this way the system is divided into four disjoint regions: (I) slow reactions with low-abundance reactants, (II) slow reactions with high-abundance reactants, (III) fast reactions with low-abundance reactants, and (IV) fast reactions with high-abundance reactants. Then an appropriate simulation method is chosen for each region ${ }^{22,40,46}$. We follow the strategy proposed Liu et al. ${ }^{22}$ where the dynamics of all mRNAs (region I) is simulated by SSA, and the other three regions (II, III, and IV) are modeled with ODEs. We shall refer to this partitioning as the 'Liu strategy'. We notice that the partitioning thresholds in Liu strategy are predefined and static. That is, while it is not guaranteed, the fast and slow sets are assumed to remain the same during the simulation.

In order to demonstrate the scale difference in our partitioning strategy, we approximate the propensity function of every reaction by its corresponding rate law function (obtaining a stochastic model with 145 reactions) and track the firing frequency of each of these reactions in a test run of Gillespie's SSA. Of 18 million reactions fired in one cell cycle, only about 34,000 (0.2\%) involve mRNA turnover, and $99.8 \%$ represent fast reactions of protein post-translational modifications. Based on this test run, we estimate that our HR hybrid scheme will run at least 100 times faster than a brute-force Gillespie simulation of a fully stochastic model. In section 3 we show that our hybrid stochastic model, using the Liu strategy, still generates accurate results that agree well with experimental observations.

Algorithm 1. Proposed in Liu et al. ${ }^{22}$ describes the hybrid ODE/SSA algorithm adopted in this paper, which is a variant of the original HR hybrid method ${ }^{46}$. Our hybrid stochastic simulation code that implements
Algorithm 1 (a FORTRAN file) is available in Supplementary Code. Algorithm 1 Hybrid Stochastic Simulation Algorithm $\operatorname{HYBRID}\left(\mathcal{R}_{\text {fast }}, \mathcal{R}_{\text {slow }}\right)$

- $t \leftarrow 0$

- While $t<T$ do

- Calculate the propensity function, $a_{i}$, for all reactions in slow subset $i=1, \ldots, k$.

- Calculate total propensity function: $a_{0}(\mathbf{s}, t)=\sum_{j=1}^{k} a_{j}(\mathbf{s}, t)$.

- Generate two uniform random variables $r_{1}$ and $r_{2}$ in $\mathrm{U}(0,1)$.

- Integrate the ODE system until an event occurs at time $t+\tau$ such that $\int_{t}^{t+\tau} a_{0}(\mathbf{s}, x) \mathrm{d} x+\ln \left(r_{1}\right)=0$.

- Select the smallest $\mu$ such that: $\sum_{i=1}^{\mu} a_{i}(\mathbf{s}, t)>r_{2} a_{0}(\mathbf{s}, t)$

- Update the state variables according to $\mu_{\text {th }}$ reaction in $\mathcal{R}_{\text {slow. }}$

- end While

Hybrid stochastic simulation algorithm: Consider a well-stirred system with $N$ species in a set $\mathcal{S}$ that interact with each other through $M$ reaction channels in a set $\mathcal{R}$. The reactions in $\mathcal{R}$ are partitioned into two disjoint subsets of fast and slow reactions denoted by $\mathcal{R}_{\text {fast }}$ and $\mathcal{R}_{\text {slow, }}$ respectively. The subset $\mathcal{R}_{\text {slow }}$ includes $k$ reactions, which are simulated using SSA, while the remaining $M-k$ fast reactions in $\mathcal{R}_{\text {fast }}$ are governed by ODEs. Let $a_{j}(\mathbf{s}, t)$ be the propensity function of the $j$-th reaction in $\mathcal{R}_{\text {slow, where } \boldsymbol{s}=}$ $\left(S_{1}(t), \ldots, S_{N}(t)\right)$ is the state vector with each element $S_{i}(t)$ representing the number of molecules of species $i$ at time $t$. In addition, let $\boldsymbol{v}_{j}=\left(v_{j 1}, \ldots, v_{j N}\right)$ be the state-change vector of the $j$-th reaction, where $v_{j i}$ denotes the change in the population of species $i$ when reaction $j$ fires. Let $\tau$ be the jump interval to the next slow reaction and $\mu$ be the index of the reaction that fires. The algorithm only needs to simulate the firings of slow reactions, while integrating the fast subset of ODEs simultaneously in Eq. (6). When a slow reaction fires, the corresponding state variables are updated. In this way the hybrid algorithm generates trajectories of state variables as the system proceeds in time. More details on implementation can be found in refs ${ }^{60-62}$.

\section{Reporting summary}

Further information on experimental design is available in the Nature Research Reporting Summary linked to this article.

\section{DATA AVAILABILITY}

Supplementary information includes two files: one for the Supplementary Text and one for the Supplementary Code. In the Supplementary Text, we present more details for the cell-cycle model used in this paper. Tables S1-S4 in Supplementary Text list the time-dependent variables, differential equations, reactions and propensity functions, and parameter values. In Table S5 in Supplementary Text we compare simulation results of 122 mutant strains with the observed phenotypes in experiment. The Supplementary Code file includes our hybrid model code in FORTRAN and statistical analysis code in MATLAB. The experimental datasets used in Fig. 2 and Fig. 4 a are available from the corresponding author upon request.

\section{CODE AVAILABILITY}

The datasets generated and analyzed during the current study are reproducible using the FORTRAN and MATLAB codes provided in the Supplementary Code.

Received: 18 July 2019; Accepted: 14 February 2020; Published online: 27 March 2020

\section{REFERENCES}

1. Borisuk, M. T. \& Tyson, J. J. Bifurcation analysis of a model of mitotic control in frog eggs. J. Theor. Biol. 195, 69-85 (1998).

2. Novak, B. \& Tyson, J. J. Modeling the cell division cycle: M-phase trigger, oscillations, and size control. J. Theor. Biol. 165, 101-134 (1993).

3. Novak, B. \& Tyson, J. J. Quantitative analysis of a molecular model of mitotic control in fission yeast. J. Theor. Biol. 173, 283-305 (1995).

4. Sveiczer, A., Tyson, J. J. \& Novak, B. A stochastic, molecular model of the fission yeast cell cycle: role of the nucleocytoplasmic ratio in cycle time regulation. Biophys. Chem. 92, 1-15 (2001). 
5. Tyson, J. J. \& Novak, B. Regulation of the eukaryotic cell cycle: molecular antagonism, hysteresis, and irreversible transitions. J. Theor. Biol. 210, 249-263 (2001).

6. Li, F., Long, T., Lu, Y., Ouyang, Q. \& Tang, C. The yeast cell-cycle network is robustly designed. Proc. Natl Acad. Sci. USA 101, 4781-4786 (2004).

7. Murray, A. W. Recycling the cell cycle: cyclins revisited. Cell 116, 221-234 (2004).

8. Jorgensen, P. \& Tyers, M. How cells coordinate growth and division. Curr. Biol. 14, 1014-1027 (2004).

9. Tyson, J. J. \& Novak, B. Temporal organization of the cell cycle. Curr. Biol. 18 759-768 (2008)

10. Tyson, J. J. Modeling the cell division cycle: $\mathrm{cdc} 2$ and cyclin interactions. Proc. Nat/ Acad. Sci. USA 88, 7328-7332 (1991).

11. Chen, K. C. et al. Integrative analysis of cell cycle control in budding yeast. Mol. Biol. Cell 15, 3841-3862 (2004).

12. Kraikivski, P., Chen, K. C., Laomettachit, T., Murali, T. \& Tyson, J. J. From START to FINISH: computational analysis of cell cycle control in budding yeast. npj Syst. Biol. Appl. 1, 15016 (2015).

13. Bornholdt, S. Boolean network models of cellular regulation: prospects and limitations. J. R. Soc. Interface 5, 85-94 (2008).

14. Davidich, M. I. \& Bornholdt, S. Boolean network model predicts cell cycle sequence of fission yeast. PLoS ONE 3, e1672 (2008).

15. Singhania, R., Sramkoski, R. M., Jacobberger, J. W. \& Tyson, J. J. A hybrid model of mammalian cell cycle regulation. PLoS Comput. Biol. 7, e1001077 (2011).

16. Okabe, Y. \& Sasai, M. Stable stochastic dynamics in yeast cell cycle. Biophys. J. 93, 3451-3459 (2007).

17. Braunewell, S. \& Bornholdt, S. Superstability of the yeast cell-cycle dynamics: ensuring causality in the presence of biochemical stochasticity. J. Theor. Biol. 245 638-643 (2007)

18. Ge, H., Qian, H. \& Qian, M. Synchronized dynamics and non-equilibrium steady states in a stochastic yeast cell-cycle network. Math. Biosci. 211, 132-152 (2008).

19. Fauré, A. et al. Modular logical modelling of the budding yeast cell cycle. Mol. BioSyst. 5, 1787-1796 (2009).

20. Kar, S., Baumann, W. T., Paul, M. R. \& Tyson, J. J. Exploring the roles of noise in the eukaryotic cell cycle. Proc. Natl Acad. Sci. USA 106, 6471-6476 (2009).

21. Ball, D. A. et al. Stochastic exit from mitosis in budding yeast: model predictions and experimental observations. Cell Cycle 10, 999-1009 (2011).

22. Liu, Z. et al. Hybrid modeling and simulation of stochastic effects on progression through the eukaryotic cell cycle. J. Chem. Phys. 136, 034105 (2012).

23. Laomettachit, T., Chen, K. C., Baumann, W. T. \& Tyson, J. J. A model of yeast cellcycle regulation based on a standard component modeling strategy for protein regulatory networks. PLOS ONE 11, e0153738 (2016).

24. Zhang, Y. et al. Stochastic model of yeast cell-cycle network. Phys. D Nonlinear Phenomena 219, 35-39 (2006).

25. Barik, D., Baumann, W. T., Paul, M. R., Novak, B. \& Tyson, J. J. A model of yeast cellcycle regulation based on multisite phosphorylation. Mol. Syst. Biol. 6, 405 (2010)

26. Barik, D., Ball, D. A., Peccoud, J. \& Tyson, J. J. A stochastic model of the yeast cell cycle reveals roles for feedback regulation in limiting cellular variability. PLoS Comput. Biol. 12, e1005230 (2016).

27. Ball, D. et al. Measurement and modeling of transcriptional noise in the cell cycle regulatory network. Cell Cycle 12, 3392-3407 (2013).

28. Di Talia, S., Skotheim, J. M., Bean, J. M., Siggia, E. D. \& Cross, F. R. The effects of molecular noise and size control on variability in the budding yeast cell cycle. Nature 448, 947-951 (2007).

29. Sveiczer, A., Tyson, J. J. \& Novak, B. A stochastic, molecular model of the fission yeast cell cycle: role of the nucleocytoplasmic ratio in cycle time regulation Biophys. Chem. 92, 1-15 (2001).

30. Steuer, R. Effects of stochasticity in models of the cell cycle: from quantized cycle times to noise-induced oscillations. J. Theor. Biol. 228, 293-301 (2004).

31. Thattai, M. \& Van Oudenaarden, A. Intrinsic noise in gene regulatory networks. Proc. Natl Acad. Sci. USA 98, 8614-8619 (2001).

32. Swain, P. S., Elowitz, M. B. \& Siggia, E. D. Intrinsic and extrinsic contributions to stochasticity in gene expression. Proc. Natl Acad. Sci. USA 99, 12795-12800 (2002).

33. Pedraza, J. M. \& Paulsson, J. Effects of molecular memory and bursting on fluctuations in gene expression. Science 319, 339-343 (2008).

34. Gillespie, D. T. A general method for numerically simulating the stochastic time evolution of coupled chemical reactions. J. Comput. Phys. 22, 403-434 (1976).

35. Chen, K. C. et al. Kinetic analysis of a molecular model of the budding yeast cell cycle. Mol. Biol. Cell 11, 369-391 (2000).

36. Sabouri-Ghomi, M., Ciliberto, A., Kar, S., Novak, B. \& Tyson, J. J. Antagonism and bistability in protein interaction networks. J. Theor. Biol. 250, 209-218 (2008).

37. Mura, I. \& Csikász-Nagy, A. Stochastic petri net extension of a yeast cell cycle model. J. Theor. Biol. 254, 850-860 (2008).

38. Bundschuh, R., Hayot, F. \& Jayaprakash, C. Fluctuations and slow variables in genetic networks. Biophys. J. 84, 1606-1615 (2003)

39. Gibson, M. A. \& Bruck, J. Efficient exact stochastic simulation of chemical systems with many species and many channels. J. Phys. Chem. A 104, 1876-1889 (2000).
40. Cao, Y., Li, H. \& Petzold, L. Efficient formulation of the stochastic simulation algorithm for chemically reacting systems. J. Chem. Phys. 121, 4059-4067 (2004).

41. McCollum, J. M., Peterson, G. D., Cox, C. D., Simpson, M. L. \& Samatova, N. F. The sorting direct method for stochastic simulation of biochemical systems with varying reaction execution behavior. Comput. Biol. Chem. 30, 39-49 (2006).

42. Li, H. \& Petzold, L. Logarithmic direct method for discrete stochastic simulation of chemically reacting systems. J. Chem. Phys. 16, 115-140 (2006).

43. Slepoy, A., Thompson, A. P. \& Plimpton, S. J. A constant-time kinetic Monte Carlo algorithm for simulation of large biochemical reaction networks. J. Chem. Phys. 128, 05 B618 (2008).

44. Gillespie, D. T. Approximate accelerated stochastic simulation of chemically reacting systems. J. Chem. Phys. 115, 1716-1733 (2001).

45. Gillespie, D. T. \& Petzold, L. R. Improved leap-size selection for accelerated stochastic simulation. J. Chem. Phys. 119, 8229-8234 (2003).

46. Haseltine, E. L. \& Rawlings, J. B. Approximate simulation of coupled fast and slow reactions for stochastic chemical kinetics. J. Chem. Phys. 117, 6959-6969 (2002).

47. Rao, C. V. \& Arkin, A. P. Stochastic chemical kinetics and the quasi-steady-state assumption: application to the gillespie algorithm. J. Chem. Phys. 118, 4999-5010 (2003).

48. Salis, H. \& Kaznessis, Y. Accurate hybrid stochastic simulation of a system of coupled chemical or biochemical reactions. J. Chem. Phys. 122, 054103 (2005).

49. Hasenauer, J., Wolf, V., Kazeroonian, A. \& Theis, F. J. Method of conditional moments $(\mathrm{mcm})$ for the chemical master equation. J. Math. Biol. 69, 687-735 (2014).

50. Cardelli, L., Kwiatkowska, M. \& Laurenti, L. A stochastic hybrid approximation for chemical kinetics based on the linear noise approximation. In Bartocci, E., Lio, P. \& Paoletti, N. (eds) Computational Methods in Systems Biology. CMSB 2016. Lecture Notes in Computer Science, vol 9859 (Springer, Cham, 2016).

51. Ghaemmaghami, S. et al. Global analysis of protein expression in yeast. Nature 425, 737-741 (2003).

52. Epstein, C. \& Cross, F. Genes that can bypass the CLN requirement for saccharomyces cerevisiae cell cycle START. Mol. Cell. Biol. 14, 2041-2047 (1994).

53. Wijnen, H. \& Futcher, B. Genetic analysis of the shared role of CLN3 and BCK2 at the G1-S transition in saccharomyces cerevisiae. Genetics 153, 1131-1143 (1999).

54. Cross, F. R. Two redundant oscillatory mechanisms in the yeast cell cycle. Dev. Cell 4, 741-752 (2003).

55. Miller, $C$. et al. Dynamic transcriptome analysis measures rates of mRNA synthesis and decay in yeast. Molecular systems biology 7, 458 (2011).

56. Di Talia, S. et al. Daughter-specific transcription factors regulate cell size control in budding yeast. PLoS Biol. 7, e1000221 (2009).

57. Laabs, T. L. et al. ACE2 is required for daughter cell-specific G1 delay in saccharomyces cerevisiae. Proc. Natl Acad. Sci. USA 100, 10275-10280 (2003).

58. Miller, M. E. \& Cross, F. R. Mechanisms controlling subcellular localization of the G1 cyclins $C \ln 2 \Delta$ and $C \ln 3 \Delta$ in budding yeast. Mol. Cell. Biol. 21, 6292-6311 (2001)

59. Cross, F. R., Archambault, V., Miller, M. \& Klovstad, M. Testing a mathematical model of the yeast cell cycle. Mol. Biol. Cell 13, 52-70 (2002).

60. Hoops, S. et al. COPASI-a COmplex PAthway SImulator. Bioinformatics 22, 3067-3074 (2006).

61. Wang, S., Chen, M., Watson, L. T. \& Cao, Y. Efficient implementation of the hybrid method for stochastic simulation of biochemical systems. J. Micromech. Mol. Phys. 2, 1750006 (2017).

62. Chen, M., Wang, S. \& Cao, Y. Accuracy analysis of hybrid stochastic simulation algorithm on linear chain reaction systems. Bull. Math. Biol. 81, 3024-3052 (2018).

\section{ACKNOWLEDGEMENTS}

This work was partially supported by the National Science Foundation under awards CCF-0953590, CCF-1526666, and MCB-1613741. Dr. Peccoud and Dr. Tyson's work was also partially supported by NIH under award GM078989.

\section{AUTHOR CONTRIBUTIONS}

M.A. and Y.C. conceived of the presented idea. M.A. developed the simulation code and performed the computations. J.P. provided the experimental data. Y.C. and J.J.T. verified the analytical methods and supervised the findings of this work. All authors discussed the results and contributed to the final manuscript.

\section{COMPETING INTERESTS}

J.P. holds an equity stake in GenoFAB Inc., a company that may benefit or may be perceived to benefit from the publication of this article. Other authors declare no conflict of interest. 


\section{ADDITIONAL INFORMATION}

Supplementary information is available for this paper at https://doi.org/10.1038/ s41540-020-0126-z.

Correspondence and requests for materials should be addressed to J.J.T. or Y.C.

Reprints and permission information is available at http://www.nature.com/ reprints

Publisher's note Springer Nature remains neutral with regard to jurisdictional claims in published maps and institutional affiliations.
Open Access This article is licensed under a Creative Commons Attribution 4.0 International License, which permits use, sharing, adaptation, distribution and reproduction in any medium or format, as long as you give appropriate credit to the original author(s) and the source, provide a link to the Creative Commons license, and indicate if changes were made. The images or other third party material in this article are included in the article's Creative Commons license, unless indicated otherwise in a credit line to the material. If material is not included in the article's Creative Commons license and your intended use is not permitted by statutory regulation or exceeds the permitted use, you will need to obtain permission directly from the copyright holder. To view a copy of this license, visit http://creativecommons. org/licenses/by/4.0/.

(c) The Author(s) 2020 